\title{
Research on a Dynamic E-commerce System based on Middleware Technology and Web Service
}

\author{
Changai Chen ${ }^{1}$, Fangfang Geng ${ }^{2}$, Jiayun Zhang ${ }^{3}$ and Yu Zhao ${ }^{4 *}$ \\ ${ }^{1}$ Department of Information Technology, Henan University of TCM, Zhengzhou, \\ Henan 450000 China \\ ${ }^{2}$ Department of Network Information Center, Henan University of TCM, \\ Zhengzhou, Henan 450000 China \\ ${ }^{3}$ Mechanical and Electrical Engineering Institute, Rizhao Polytechnic, Rizhao, \\ Shandong,276826 China \\ ${ }^{4}$ School of Computer Science and Technology, ZhouKou Normal University, \\ Zhoukou, Henan,466001 China \\ *Corresponding author: Yu Zhao,E-mail:lunwen1208@yeah.net
}

\begin{abstract}
With the continuous development of information technology and the growing popularity of the Internet, the E-commerce system based on the traditional distributed technology has exposed more and more problems and the development of dynamic Ecommerce system has become the target of the next generation of E-commerce. Middleware technology is the trend of the development of software, which is famed and the software developing fastest. So the middleware technology is used to develop the Ecommerce system in order to propose a new generation dynamic E-commerce system. Several key techniques J2EE architecture and MVC design pattern are introduced. Then a service-oriented architecture(SOA) and a service-oriented dynamic E-commerce system based on middleware technology and web services are researched and designed. Web services can provide exchange of information and system integration for dynamic Ecommerce, support more complex types of web interaction and collaboration. J2EE technology and MVC design pattern can simplify the software development, improve the software performance and quickly construct the dynamic E-commerce system with the good expansibility, maintainability, dependability and high usability.
\end{abstract}

Keywords: Dynamic E-commerce system, web services, J2EE architecture, MVC design pattern, middleware technology

\section{Introduction}

With the continuous development of information technology and the increasing popularity of Internet technology, E-commerce obtains the rapid development [1-2]. However, the E-commerce environment based on the traditional technology is lack of the unified commerce publishing and discovery mechanism, which becomes very difficult for the mutual information exchange and cooperation. At the same time, it also has shortcomings, such as long development cycle, poor reconstruction, difficulty in upgrading and maintenance. How to efficiently construct the Ecommerce application system of the enterprise and promote the E-commerce from the static E-commerce to the dynamic E-commerce have already become an urgently studied problem [3-6].

The dynamic E-commerce is an E-commerce system which can dynamically adapt to the changing business process. It can realize the dynamic integration of the application systems of different enterprises, and show the real value of E-commerce. 
In recent years, a lot of new E-commerce systems are proposed. Chen et al. [7] proposed a Java based dynamic agent infrastructure for E-Commerce automation, which supports dynamic behavior modification of agents, a significant difference from other agent platforms. Rao and Feng [8] proposed an integration web services application framework model, i.e. Web Services-oriented Dynamic E-Business Framework (WS-DEBF) model, which was based on a four-layer logic structure with application layer, resource share and information exchange layer, data resource layer and network layer. In order to improve the integration capability and expansibility in web services-based Dynamic E-Business (DEB) application. Zhang et al. [9] proposed a framework for dynamic and personalized composition of Web services using an approach that integrates not only functional attributes of Web services, but also nonfunctional attributes such as service requirements, quality of service, and the preferences and constraints of individual service consumers. Jain et al. [10] proposed a fuzzy enhanced high level petri net (FEHLPN) model in order to provide a vehicle for dynamic modelling and analysis of supply chain operations in vague and uncertain environments. The proposed model captures the capability of petri nets for graphical and analytical representation of dynamic SCNs with the management of uncertain information provided by fuzzy logic. Burn and Ash[11] proposed a dynamic model for e-business strategy derived from the results of a longitudinal analysis of enterprise resource planning (ERP) enabled organizations. The model focuses on realizing the benefits of B2B interaction through the alignment of ERP with different e-business strategies, increasing emphasis on employee empowerment and successful management of value alliances. Research limitations/implications. Li et al. [12] proposed an interactive and cooperative dynamic e-business application using grid service. Grid service is the upgrade of web service. Chen and $\mathrm{Wu}$ [13] proposed a dynamic e-commerce integration based on J2EE-enabled Web services. The dynamic discovery of the diversified business service among multi-enterprises over the Internet becomes practical by the design and prototype of Web services based on J2EE. Alexander et al. [14] studied the impact of technology, cultural, and socio-economic factors on the global diffusion of e-commerce. A cross-country regression model analyzes the determinants of ecommerce diffusion and the results used in a cluster analysis to provide further evidence that the propensity for e-commerce depends on the interplay among the different factors. Netessine et al. [15] focused on two practical business settings: with (the Emergency Replenishment Model) and without (the Lost-Sales Model) the possibility of inventory replenishment in the case of a product stock out. Ulmer and Tao, [16] proposed a novel scheme based on Web services and middleware technologies to support delayed transactions, which are critical in e-business marketplaces like e-auction or scenarios where delaying a transaction may increase the value proposition to the human user. Li and Iijima[17] proposed a situation calculus based approach for dynamically managing e-Business services in the ubiquitous environment to respond to customers in a rapid and intelligent way. The approach can implement the functions of service automatic composition and model verification. Chen et al. [18] proposed an organization structure-based access control (OSAC) model based on a task-role-based access control (T-RBAC) model. The proposed model extends the concepts of static separation of duty (SSD), dynamic separation of duty (DSD), prerequisite. Yuan and Lu [19] proposed an architecture of adaptive integration of EDA and SOA based on the novel concept of value-centric processing and communication of events grounded on value theory. This architecture has also been used on a case study of distributed care service delivery. In the architecture, value models are devised to represent events consumed and develop a value-centric mechanism for this adaptive integration. Nabi [20] proposed a secure framework method based on existing techniques that treats 
security as a first-class concept and considers its interaction with business logic. Rallapalli [21] proposed an alternate approach to effectively address the way privacy agreements for initiating through web services. In this new framework, the consumer and the service provider can mutually negotiate on the privacy terms. Li and Wang [22] proposed a trust cloud-based dynamic secure trust management model (TCDSTM) for the trust evaluation problem in P2P e-commerce. Cloud theory was used to describe the trust and the trust level in TCDSTM. Cheng [23] proposed perishable products demand state model and dynamic bundling decision process model based on delay buying utility analysis, and then gave the stochastic parameters estimation method for the decision process model. Xie et al. [24] proposed an operation scheme based on dynamic e-business service system and a diversified profit-making mode. Zhang et al. [25] proposed a Dynamic Scanning approach for XML structural clustering based on Trie Matching (DSTM). The extensive experiments on XML documents in e-business is conducted in order to demonstrate the effectiveness and efficiency of DSTM. The experimental results show that DSTM is approximately linear to the size of XML documents. In addition, the other new methods are proposed in the recent years [26-31].

Web service provides a new way to achieve business objectives, run and build business and find new applications for managing business, in order to provide a technical for constructing a dynamic e-commerce platform. Web service is a dynamic interactive software component based on open standards, Intranet, and Internet based on industry standards, and it is easy to implement the low risk technology. The application of web service in the enterprise mainly to be able to integrate different manufacturers, different hardware and different languages. At the same time, it can provide special assistance to those enterprises that have formed by mergers and acquisitions. Middleware is a kind of software between the application software and system software. It is independent of hardware or database vendor, and the connection between client and service. A good middleware should be able to solve all kinds of application interconnection problems. The communication ought to support a variety of communication protocols, various communication services model, the transmission of various data content, data format translation, flow control, data encryption and data compression. The core of middleware is to solve these problems of name service, security control, concurrency control, reliability and other aspects of the guarantee. So the middleware technology is used to develop the E-commerce system in order to propose a new generation dynamic Ecommerce system. Then a service-oriented architecture(SOA) and a service-oriented dynamic E-commerce system based on middleware technology and web services are researched and designed in this paper.

The rest of this paper is organized as follows. Section 2 briefly introduces service oriented architecture(SOA) and web services. Section 3 briefly introduces middleware technologies, including J2EE technology and MVC design pattern. Section 4 proposes a novel dynamic E-commerce system based on middleware technology and web services. Finally, the conclusions are discussed in Section 5.

\section{Service Oriented Architecture(SOA) and Web Services}

\subsection{SOA}

Service oriented architecture(SOA) is used to construct the loosing coupling software system to publish commerce functions in the manner of programmable and accessible services. The key conception of SOA is SERVICES, each application of SOA is regarded as service in order to call and manage. The SOA defines the design principles:

(1) The functions are divided into the less and reused model by using modularization. 
(2) The clients and servers of the loosing coupling do not require the close dependence.

(3) The encapsulation encloses function modularization.

W3C defined the SOA: the provider of the service provides the ultimate results for users by accomplishing a set of work. On a certain extent, the SOA is a model, which is used to design, develop, deploy and manage the discrete logic units in computer environment.

The SOA has three roles of service broker, service provider and service requester. The architecture of SOA is show in Figure 1.

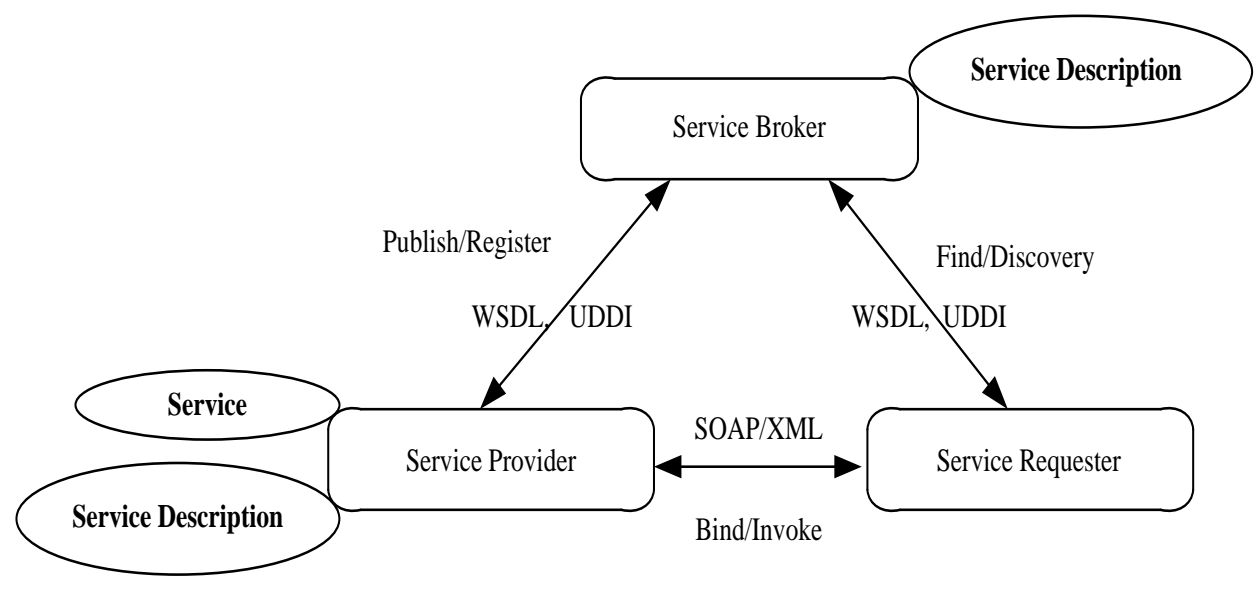

Figure 1. The SOA Architecture

In the Figure 1, the service broker registers and makes the classifications of the published service providers. The service requester seeks the requisite services by using services broker, and makes use of the services. The service provider publishes its own services, and also makes response to the request. The components of SOA have one or more roles. These roles execute finding operation, publishing operation and binding operation. The finding operation is to help the service requester to find out the special services help by using Service broker. The publishing operation is to help the service provider to register its own function and interface. The binding operation is to help the service requester to use the provided services in deed. So the service is a basic task in the SOA and it lies in abstract layer between the commerce requirement and bottom technology. In the process of designing the system, the dynamic relation between the function requirements and acquired services, the relation between the services and bottom technology of the services are understood. Then the basic services must meet the requirement of definition definitude and function singularity in the SOA. So each single deployment services often rely on the else application system.

\subsection{Web Service}

Web service is a class of general service from the Internet. It uses Extensible Markup Language(XML) to receive or send the message and is not constrained by any operating system or programming language. The web service provides a new development model for establishing distributed Web application. The wide popularization, simplicity and platform neutrality of Web service has brought incomparable advantages of SOA to promote the rapid development of SOA technology and provide a good technology for system integration based SOA. Under the guidance of SOA, the enterprise informatization construction is to develop enterprise resources according to the reasonable size into service, then the business process of enterprise is constructed by the service choreography. At the same time, the legacy of the enterprise information system is packaged into service 
to participate in the business process in order to provide the enough flexibility for dealing with the business process reengineering because of market changes, managing enterprise IT resources, providing a good environment and improving the utilization efficiency.

Web service combines the advantages of component-oriented methods and web techniques. It can publish, locate and transfer modularized application in the web. The provided functions of Web service may be simple, but they contain the extraordinary complicated business logic. Once Web service is deployed, the other applications can find and request them. The key techniques of Web service are shown in Figure 2.

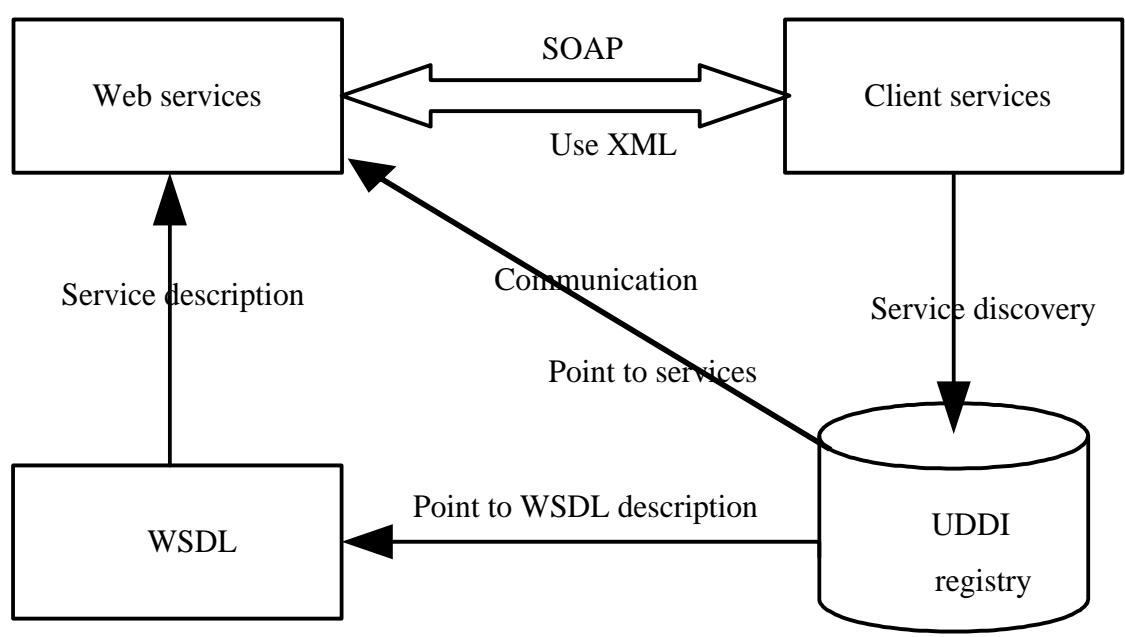

Figure 2. Key Techniques of Web Services

As can be seen from Figure 2, the kernel of Web service is service. Web service represents a kind of implementation of SOA, and they are the most popular one. In addition, when the components of SOA interact, three operations in the SOA can process. So some standardized techniques are used in Web service, such as Universal Description, Discovery and Integration (UDDI), Web Services Description Language(WSDL), Hyper Text Transfer Protocol(HTTP), Simple Object Access Protocol(SOAP), and XML and so on. Web service is becoming the best selection to develop application of SOA.

\section{Middleware Technologies}

\subsection{J2EE Architecture}

J2EE is an architecture based on Java platform to simplify the development, deployment and management. It consolidates these advantages of the standard, JDBC, JNDI, RMI, CORBA API. And it also provides the protected data security pattern in Internet applying and comprehensively supports EJB, Java Servlet, JTA, JTS, JavaMail and XML technologies. Multi-layer enterprise application model based on J2EE technology is divides into four layers:

(1) Client layer is application program client based on web way or the traditional way.

(2) Web layer generally includes JSP page or Servlet.

(3) Business logic layer mainly points to EJB in J2EE architecture and EJB processes the code logic in business layer.

(4) Enterprise information system layer includes enterprises infrastructure equipments, such as database system and other left behind information system.

J2EE architecture is shown in Figure 3. 


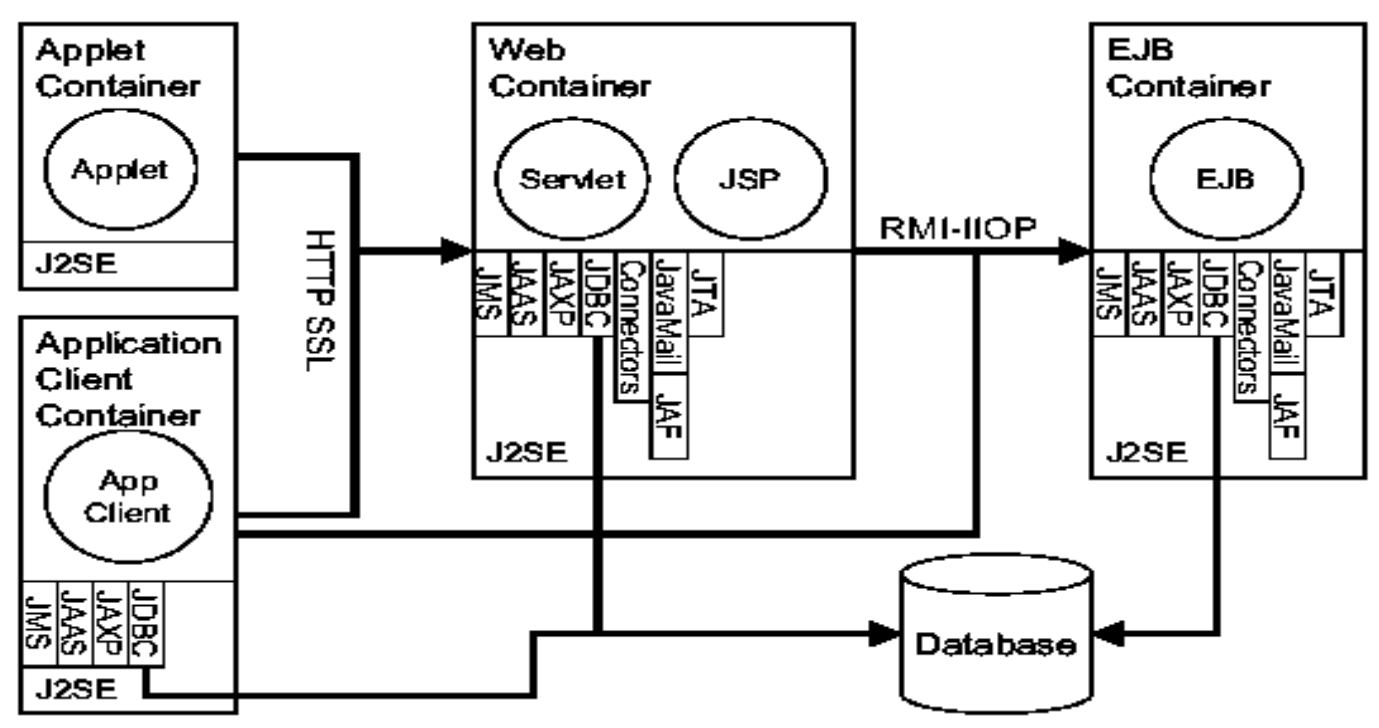

Figure 3. J2EE Architecture

J2EE provides a variety of developing technologies, the more commonly used technologies are described as follows:

(1) JSP technology is a new dynamic web application technology standard. JSP web page is composed of the traditional HTML web page files, which are inserted Java program files and JSP tags. Consequently, it comes into being a dynamic page on the server according to the client request.

(2) Servlet is a small Java program on the server side and it can realize HttpServlet interface. It can respond and deal with the client request by using Servlet API, and even it can bring a dynamic HTML page.

(3) EJB provides a framework for developing and implementing the distributed business logic. So it is very significant to simplify the development of the scalability and the highly complex enterprise application. The EJB standard defines EJB component when and how to interact with their containers. The containers provide public services, such as directory services, business management, security, resource buffer pool, fault tolerance and so on.

\subsection{MVC Design Pattern}

Model-View-Controller (MVC) is a software design pattern. The MVC) is divided into three modules, each module has own function. There are three categories in the following:

(1) The model is the principal part of application program. The model expresses business data, it is clearly said that one model is a record in database. One model may provide the data for many views, in this way it reduces the repeated code for application program.

(2) The view is an interrelated part of User Interface (UI) in application program. One of advantages is that it can process many different views for application program by using MVC. The view is only regarded as a means of output data and user operation.

(3) The controller controls UI data displaying and updates the model object state according to the users' input. The controller accepts the user input and calls the model and view to complete the user demand. So when the hyperlinks is clicked in the web page and HTML table list is sent, the controller does not have any output and make any processing. It only receives the request and determines calling which model to deal with the request, and it confirms to use which view to display the returning data of the model processing. There is the relationship of the model, the view and the controller, shown as Figure 4. 


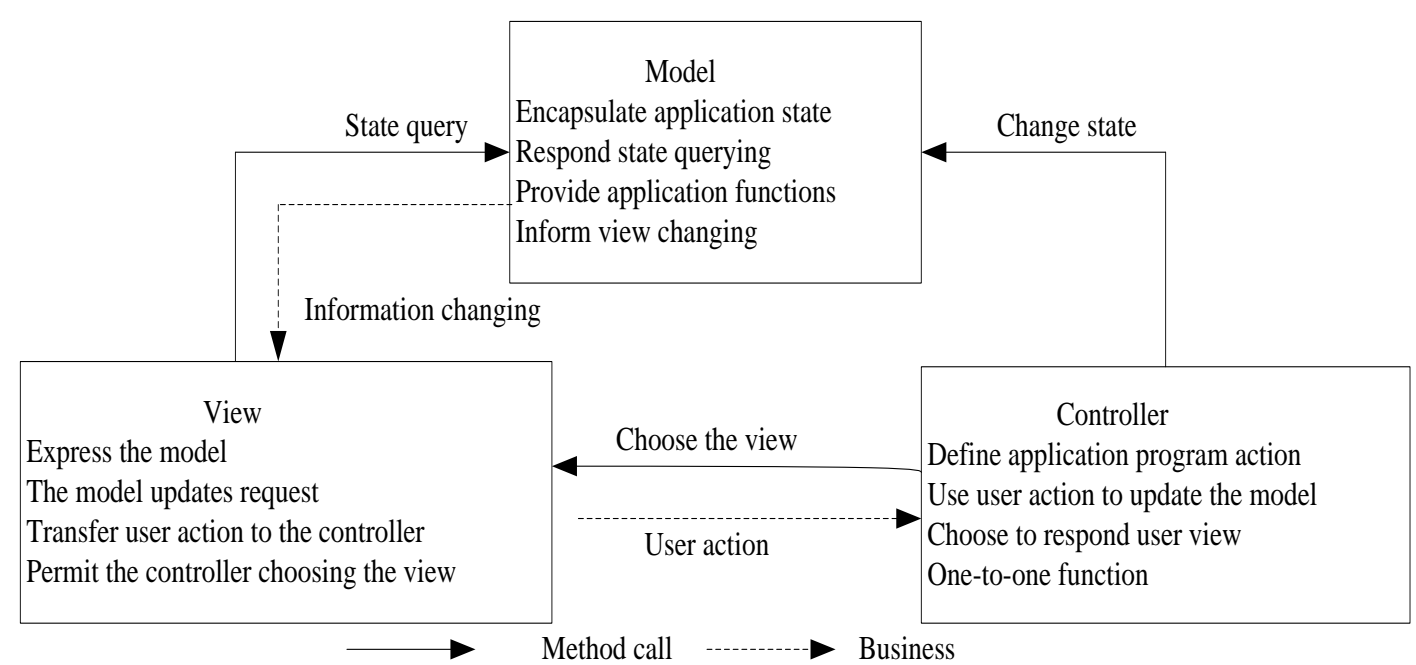

Figure 4. MVC Design Pattern

\section{Dynamic E-commerce System based on Middleware Technology and Web Services}

Because the traditional E-commerce system is short of the nicer integrated ability, and exists the large quantity expense due to the commerce flow changing, it can effectively solve the customizing demand of each category to users. So the dynamic E-commerce system is an optimal scheme for solving the existing problems of the traditional Ecommerce system. The dynamic E-commerce system is the next generation E-commerce, which emphasizes the comprehension and infrastructure of the B2B. It can effectively create the best benefit to the interior and exterior enterprises by adjusting the Internet standard and general infrastructure. The dynamic E--commerce system architecture is shown in Figure 5.

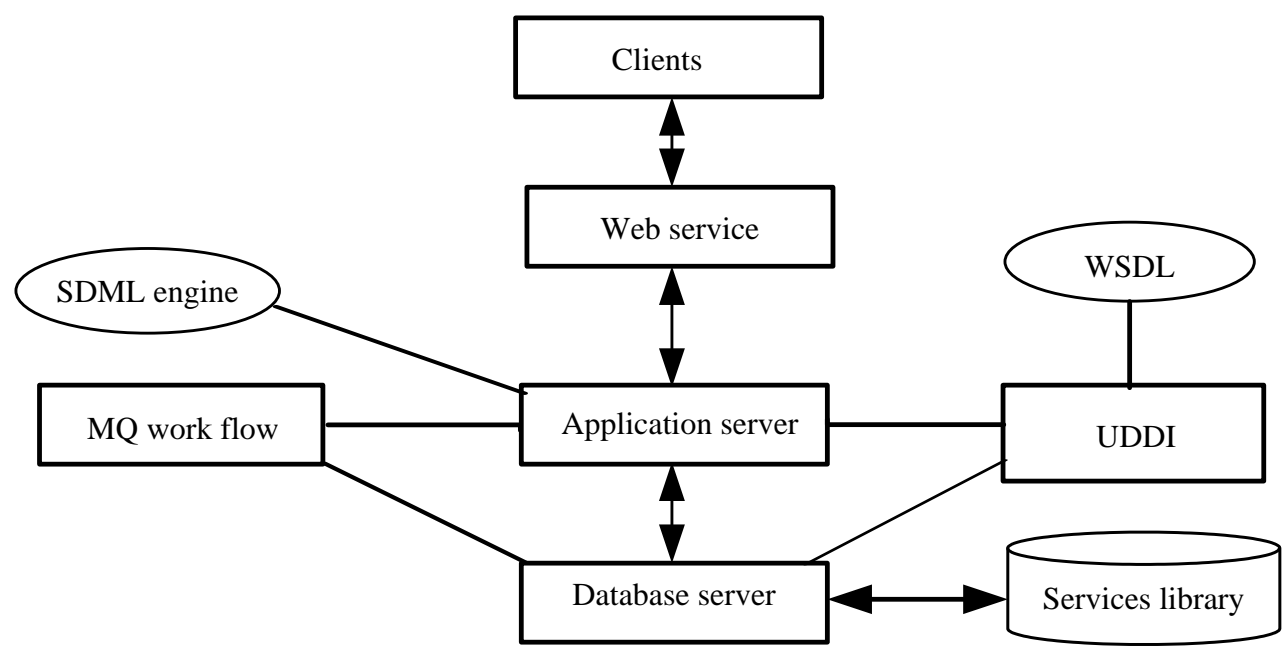

Figure 5. Dynamic E-commerce System Architecture

This dynamic E-commerce system architecture exists some advantages, such as the relative independence, convenient operation and so on. But it also exists some disadvantages, such as too substantial investment, different data sharing, discommodious information exchange, long development time and so on. At the same time, due to the 
dynamic nature of E-commerce, the provider of web service is constantly changing, and each type of members provides the web service which defines the unified service interface by the information coordination center. The service provider uses the developed dynamic connection mode to dynamically obtain the provider of web service, then call the service one by one. So a novel dynamic E-commerce system based on middleware technology and web service is proposed in this paper. The novel dynamic E-commerce system architecture based on middleware technology and web service is shown in Figure 6.

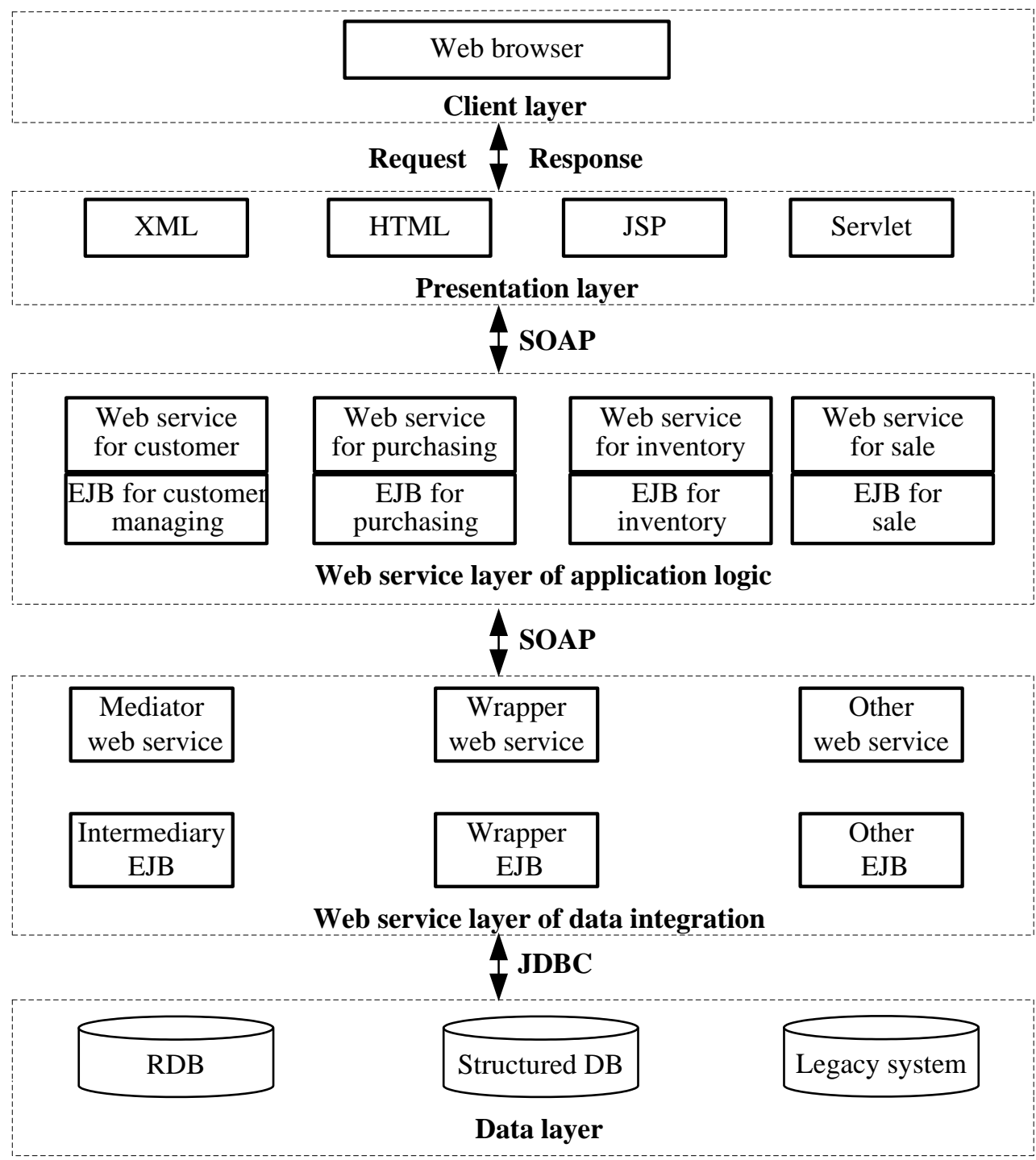

Figure 6. The Novel Dynamic E-commerce System Architecture

J2EE server is the core of novel dynamic E-commerce system architecture. It includes web service layer of data integration, web service layer of application logic and presentation layer. The web service layer of data integration uses the mediator/wrapper integration method to achieve various data resources of the wrapper and mediator and complete decomposing the global query and translating into each data source model by using EIB components. Each EJB component is deployed as web service. When application logic need to access the data, the SOAP is used to call the corresponding web service. The web service layer of application logic is often said the business layer. It can realize purchasing management, customer management, inventory management and sale management by using EIB components. And it is deployed as web service. The 
presentation layer assembles various web service to complete dynamic content display and process logic control, establish distributed E-commerce application system by using JSP and Servlet and so on. Each web user can call by using the browser. Web server is an important part of J2EE architecture. The main technologies are Servlet and JSP. Its function implements UI. Because the essential of JSP and Servlet is a small Java program on the server side, it can exchange Java Applet and HTML with the client. Not only is implementing speed fast and is UI generation flexible, but also is the security very good, at the same time, it can realize some simple application logic. J2EE provides all needed services of the distributed computing component in the EJB server, such as life-cycle management of the component, database connectivity management, distributed business service, automatic fault tolerance and laden automatic equilibrium capability and so on. So the proposed system architecture can realize the dynamic E-commerce system with loose coupling.

\section{Conclusion}

The dynamic E-commerce is the goal of E- commerce development, and web services and middleware technology are their key techniques. Web service uses Extensible Markup Language(XML) to receive or send the message and is not constrained by any operating system or programming language. It provides a new method to achieve business objectives, run and build business and find new applications for managing business. Middleware is a kind of software between the application software and system software. It is independent of hardware or database vendor, and the connection between client and service. A good middleware should be able to solve all kinds of application interconnection problems. The core of middleware is to solve these problems of name service, security control, concurrency control, reliability and other aspects of the guarantee. So the middleware technology is used to develop the E-commerce system in order to propose a new generation dynamic Ecommerce system. Several key techniques J2EE architecture and MVC design pattern are introduced. Then a service-oriented architecture(SOA) and a service-oriented dynamic Ecommerce system based on middleware technology and web service are researched and designed. In the proposed dynamic E-commerce system architecture, the provider of web service is constantly changing, and each type of members provides the web service which defines the unified service interface by the information coordination center. The service provider uses the developed dynamic connection mode to dynamically obtain the provider of web service, then call the service one by one. As a result, it brings enormous benefits for the commercial integration.

\section{Acknowledgments}

This research was supported by Key Scientific Research Project of Higher Education of Henan Province: Development and application of MOOC under the background of higher education informatization (project number:16B520016), the Soft Science Project of Science Department of Henan Province: The research of student assessment system rationality about local universities (project number: 142400411267).

\section{References}

[1] S. Park, J. M. Choi and H. Y. Yoo, "Integrated model of service-oriented architecture and web-oriented architecture for financial software", Journal of Information Science and Engineering, vol. 28, no. 5, (2012), pp. 925-939.

[2] H. Liao, R. W. Proctor and G. Salvendy, "Content preparation for cross-cultural e-commerce: A review and a model", Behaviour and Information Technology, vol. 27, no. 1, (2008), pp. 43-61. 
[3] M. Á. Conde, F. G. Peñalvo, M. Alier, E. Mayol and C. F. Llamas, "Implementation and design of a service-based framework to integrate personal and institutional learning environments", Science of Computer Programming, vol. 88, (2014), pp. 41-53.

[4] D. K. Gangeshwer, "E-commerce or internet marketing: A business review from Indian context", International Journal of u- and e- Service, Science and Technology, vol. 6, no. 6, (2013), pp. 175-182.

[5] S. S. Li and E. Karahanna, "Online recommendation systems in a B2C E-commerce context: A review and future directions", Journal of the Association of Information Systems, vol. 16, no. 2, (2015), pp. $72-$ 107.

[6] W. Wresch and S. Fraser, "Managerial strategies used to overcome technological hurdles: A review of E-commerce efforts used by innovative caribbean managers", Journal of Global Information Management, vol. 14, no. 3, (2006), pp. 1-16.

[7] Q. M. Chen, M. C. Hsu, U. Dayal and M. Griss, "Multi-agent cooperation, dynamic workflow and XML for E-Commerce automation", HP Laboratories Technical Report, vol. 136, (1999), pp. 1-6.

[8] Y. Rao and B. Q. Feng, "Web service-oriented dynamic E-business integration framework", Computer Integrated Manufacturing Systems, CIMS, vol. 10, no. 11, (2004), pp. 1454-1458.

[9] D. S. Zhang, M. Chen and L. N. Zhou, "Dynamic and personalized web services composition in Ebusiness", Information Systems Management, vol. 22, no. 22, (2005), pp. 50-65.

[10] V. Jain, S. Wadhwa and S. G. Deshmukh, "e-Commerce and supply chains: Modelling of dynamics through fuzzy enhanced high level petri net", Sadhana - Academy Proceedings in Engineering Sciences, vol. 30, no. 2-3, (2005), pp. 403-429.

[11] J. Burn and C. Ash, "A dynamic model of e-business strategies for ERP enabled organisations", Industrial Management and Data Systems, vol. 105, no. 8, (2005), pp. 1084-1095.

[12] F. F. Li, D. Y. Qi, Z. L. Zhang, L. M. Zhang and Y. Q. Xu, "A novel dynamic e-business system based on grid technology", Journal of Computational Information Systems, vol. 1, no. 3, (2005), pp. 485-490.

[13] T. B. Chen and W. Wu, "Design and implementation for dynamic e-commerce integration based on J2EE-enabled Web services", Journal of Dalian University of Technology, vol. 45, no. 5, (2005), pp. 749-755.

[14] Y. Alexander, D. Jayoti, B. John and C. Kathryn, "A composite-model for E-commerce diffusion: Integrating cultural and socio-economic dimensions to the dynamics of diffusion", Journal of Global Information Management, vol. 14, no. 3, (2006), pp. 17-38.

[15] S. Netessine, S. Savin and W. Q. Xiao, "Revenue management through dynamic cross selling in Ecommerce retailing", Operations Research, vol. 54, no. 5, (2006), pp. 893-913.

[16] D. B. Ulmer and L. X. Tao, "Architectural solutions to agent-enabling e-commerce portals with pull/push abilities", WSEAS Transactions on Computers, vol. 5, no. 5, (2006), pp. 1026-1033.

[17] B. Li and J. Iijima, "A situation calculus based approach to dynamic management of e-business services", Journal of Systems Science and Systems Engineering, vol. 16, no. 3, (2007), pp. 336-355.

[18] T. Y. Chen, Y. M. Chen, C. B. Wang, H. C. Chu, "Flexible authorisation in dynamic e-business environments using an organisation structure-based access control model", International Journal of Computer Integrated Manufacturing, vol. 22, no. 3, (2009), pp. 225-244.

[19] S. T. Yuan and M. R. Lu, "An value-centric event driven model and architecture: A case study of adaptive complement of SOA for distributed care service delivery", Expert Systems with Applications, vol. 36, no. 2, (2009), pp. 3671-3694.

[20] F. Nabi, "Designing a framework method for secure business application logic integrity in e-commerce systems", International Journal of Network Security, vol. 12, no. 1, (2011), pp. 29-41.

[21] M. V. Rallapalli, "A privacy agreement negotiation model in B2C e-commerce transactions", International Journal of Information Security and Privacy, vol. 5, no. 4, (2011), pp. 1-7.

[22] Z. Y. Li and R. C. Wang, "Dynamic secure trust management model for P2P e-commerce environments", Journal on Communications, vol. 32, no. 3, (2011), pp. 50-59.

[23] Y. Cheng, "Delay buying behavior-oriented perishable products dynamic bundling strategy in ecommerce setting", System Engineering Theory and Practice, vol. 31, no. 10, (2011), pp. 1892-1902.

[24] P. S. Xie, Z. Y. Rui and J. Cao, "An operation scheme and profit-making mode based on dynamic Ebusiness service system and diversified benefit for MISP", International Journal of Digital Content Technology and its Applications, vol. 6, no. 6, (2012), pp. 190-199.

[25] J. Zhang, H. Q. Zhu and G. Sun, "A dynamic scanning approach based on trie matching for clustering XML data in E-business", Communications in Computer and Information Science, vol. 332, (2013), pp. 715-728.

[26] A. A. Costa and L. V. Tavares, "Social e-business as support for construction e-procurement: Eprocurement network dynamics", Automation in Construction, vol. 43, (2014), pp. 180-186.

[27] P. Moradi and S. Ahmadian, "A reliability-based recommendation method to improve trust-aware recommender systems", Expert Systems with Applications, vol. 42, no. 21, (2015), pp. 7386-7398.

[28] S. S. Xiao and M. Dong, "Hidden semi-Markov model-based reputation management system for online to offline (O2O) e-commerce markets", Decision Support Systems, vol. 77, (2015), pp. 87-99.

[29] Z. B. Zheng, M. R. S. Lyu, H. M. Wang, "Service fault tolerance for highly reliable service-oriented systems: an overview", Science China Information Sciences, vol. 58, no. 5, (2015), pp. 1-8. 
[30] W. L. Geng and J. Tan, "Construction and simulation of competitiveness evaluating model for ecommerce enterprise based on DEA", International Journal of $\mathrm{u}$ - and e- Service, Science and Technology, vol. 8, no. 2, (2015), pp. 61-70.

[31] G. Y. Jiang, F. C. Ma, J. Shang, P. Y. K. Chau, "Evolution of knowledge sharing behavior in social commerce: An agent-based computational approach", Information Sciences, vol. 278, (2014), pp. 250266.

\section{Authors}

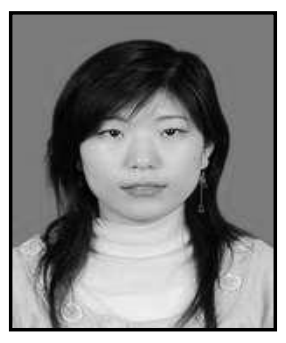

Changai Chen, she is lecturer, received the Master degree in Control Science and Engineering from Wuhan University of Technology in 2009, Wuhan, China. The main research directions: Artificial Intelligence, Computer Simulation and Software Engineering.

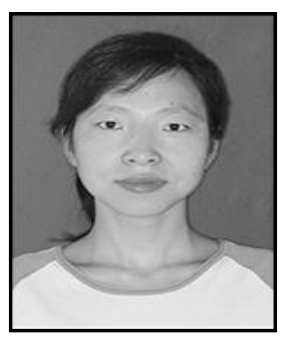

Fangfang Geng, she is a lecturer, received the Master degree in Communication and Information System from North China Electric Power University in 2009, Baoding, China. The main research directions: Information Security and Processing, Computer Network and Software Engineering.

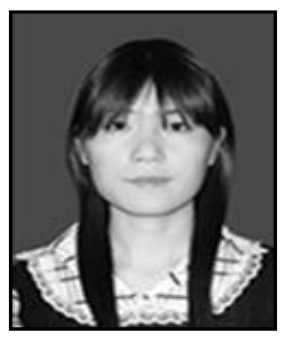

Jiayun Zhang, she is a lecturer, received the Master degree in Control Theory and Control Engineering from Changchun University of Technology, China in 2009. Her research interests include control Engineering and intelligent method

Yu Zhao, Professor, Master. The main research directions: Computer Wireless Sensor Network, Computer Application. 
International Journal of Future Generation Communication and Networking Vol. 9, No.9, (2016) 